\title{
Epitope mapping from real time kinetic studies - Role of cross- linked disulphides and incidental interacting regions in affinity measurements: Study with human chorionic gonadotropin and monoclonal antibodies
}

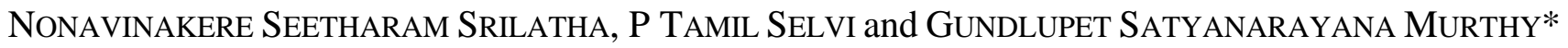 \\ Department of Molecular Reproduction, Development and Genetics, \\ Indian Institute of Science, Bangalore 560 012, India \\ *Corresponding author (Fax, 91-80-23600999; Email, gundlupetmur@indiatimes.com)
}

\begin{abstract}
Real time kinetic studies were used to map conformational epitopes in human chorionic gonadotropin (hCG) for two monoclonal antibodies (MAbs). The epitopes were identified in the regions $(\alpha 5-14$ and $\alpha 55-62)$. The association rate constant $\left(k_{+1}\right)$ was found to be altered by chemical modification of hCG, and the ionic strength of the reaction medium. Based on these changes, we propose the presence of additional interactions away from the epitope-paratope region in the hCG-MAb reaction. We have identified such incidental interacting regions (IIRs) in hCG to be the loop region $\alpha 35-47$ and $\alpha 60-84$. The IIRs contribute significantly towards the $K_{A}$ of the interaction. Therefore, in a macromolecular interaction of hCG and its MAb, $K_{A}$ is determined not only by epitopeparatope interaction but also by the interaction of the nonepitopic-nonparatopic IIRs. However, the specificity of the interaction resides exclusively with the epitope-paratope pair.
\end{abstract}

[Srilatha N S, Selvi T and Murthy G S 2005 Epitope mapping from real time kinetic studies - Role of cross-linked disulphides and incidental interacting regions in affinity measurements: Study with human chorionic gonadotropin and monoclonal antibodies; J. Biosci. 30 359-370]

\section{Introduction}

Human chorionic gonadotropin (hCG) plays a major role in human fertility and reproduction and has been one of the most widely studied gonadotropins. hCG is purified from early pregnancy urine in large bulk making it one of the easiest of the hormones available for structural investigations. Consequently hCG is extensively investigated, has high resolution X-ray crystal structure (Lapthorn et al 1994) and is much used in structure-function analysis (Purohit et al 1997; Matzuk et al 1990; Ashish et al 2002a). It has two independent subunits, the species-specific alpha $(\alpha)$ and the function-specific beta $(\beta)$, and the heterodimer consist of one of each subunits in the biologically functional dimer. It is a highly cross-linked molecule with 11 disulphide bridges ( 5 in $\alpha$-subunit and 6 in the $\beta$ subunit) imparting a rigidity to the structure, thereby rendering the epitope stable to chemical modifications, enzyme digestion and acid and alkali treatments (Venkatesh and Murthy 1997a,b). However, its structural integrity is heavily dependent on the disulphide bonds, and its reduction will completely destroys the structure (Murthy and Srilatha 1996; Murthy and Venkatesh 1996; Troalen et al 1988; Berger et al 1996). We have developed an epitopemapping strategy, based on the effect of chemical modification of hCG on its binding to monoclonal antibody (MAb) (Venkatesh et al 1995; Venkatesh and Murthy 1997a). In a dozen MAbs we have investigated, modifi-

Keywords. Association constant; epitope mapping; incidental interacting regions; kinetics; monoclonal antibody

Abbreviations used: Ac, Acetyl; $\beta$-CTP, $\beta$-c-terminal peptide; Chy, chymotrypsin; hCG, human chorionic gonadotropin; hLH, human luteinising hormone; IhCG, radiolabelled ${ }^{125}$ I human chorionic gonadotropin; IIR, incidental interacting regions; $k_{-1}$, dissociation constant; $k_{+1}$, association constant; $K_{A}$, affinity constant; MAb, Monoclonal antibody; PMSF, phenyl methyl sulphonyl fluoride; Try, trypsin; SS-SPRIA, single step solid phase radioimmunoassay. 
cation of the subunit with group-specific reagents resulted in loss of MAb-binding activity, allowing identification of the epitopic regions. However, two of our MAbs showed an anomaly. They were $\alpha$-subunit-specific with negligible binding to the $\beta$-subunit. Yet, on chemical modification of the $\alpha$-subunit the loss of activity was insignificant. Therefore, for these two MAbs epitope, mapping by chemical modification was not possible. A parameter of use to circumvent such a problem came from our studies on the real time kinetics of hCG-MAb interaction (Murthy 1996; Murthy and Venkatesh 1996b). In the following we report on the epitope analysis of these two MAbs, done by combination of chemical modification procedure and real time kinetic analysis of the dissociation of the radiolabelled IhCG-MAb complex, represented throughout as IhCG-MAb.

\section{Materials and methods}

hCG was prepared from early pregnancy urine using immunoaffinity chromatography. MAbs were obtained using native hCG as antigen (Venkatesh et al 1995). They were $\alpha$-specific MAbs and had cross reactivity of $<5 \%$ for the $\beta$-subunit.

Epitope analysis was carried out as already described using activity changes in radioimmunoassay (RIA) on chemical or enzymatic modification of hCG (Venkatesh and Murthy 1997a). Iodination of hCG was done by the standard procedure using iodogen method (Fraker and Speck 1978).

Digestion of hCG by trypsin (try) and chymotrypsin (chy) was carried out at $1 \%$ concentration of enzyme and $2 \%$ concentration of hCG. Digestion was carried out for $5 \mathrm{~h}$ at $37^{\circ} \mathrm{C}$, and terminated by adding trypsin inhibitor/ phenyl methyl sulphonyl fluoride (PMSF). The extent of hydrolysis was measured by the increase of the free amino groups after hydrolysis, and was found to be 12 and 8 respectively (Venkatesh et al 1995). Acetylation of hCG was carried out using acetic anhydride, and was found to be quantitative as determined by colour reaction.

\subsection{Determination of real time kinetic constants of the reaction}

The MAbs were immobilized on nitrocellulose (NC) discs, incubated with radiolabelled [ $\left.{ }^{125} \mathrm{IhCG}\right]$ to obtain immobilized IhCG-MAb (Srilatha and Murthy 2002). The complex was subjected to quantitative dissociation with excess of unlabelled hCG. The dissociation profile was analysed to obtain both $k_{-1}$ and $k_{+1}$ as described earlier (Ashish et al 2002b). Briefly, $k_{-1}$ was determined by the curve-fitting analysis, and $K_{A}$ by the binding data. $k_{+1}$ was obtained by equating it to $K_{A} \times k_{-1}$. $k_{-1}$ is expressed in $\min ^{-1}$, $k_{+1}$ in $\mathrm{mol}^{-1} \mathrm{~min}^{-1}$, and $K_{A}$ in mol.

\subsection{Calculation of the changes in the activation energy} $\left(\Delta \mathrm{E}_{\mathrm{a}}\right)$ at varying salt concentrations

The equation describing the chemical reaction is represented by

$$
k=Z e^{(-E a / R T)} .
$$

Therefore

$$
\begin{aligned}
& \ln k_{(0.01 \mathrm{M})}=\ln Z-\left(E_{0.01 \mathrm{M}} / R T\right) \text { and } \\
& \ln k_{(0.1 \mathrm{M})}=\ln Z-\left(E_{0.1 \mathrm{M}} / R T\right)
\end{aligned}
$$

where, $k_{0.1 \mathrm{M}}$ and $k_{0.01 \mathrm{M}}$ correspond to rate constants in $0.01 \mathrm{M}$ and $0 \cdot 1 \mathrm{M}$ salt concentrations.

Since change in salt concentration from $0.01 \mathrm{M}$ to $0 \cdot 1 \mathrm{M}$ does not result in loss of binding activity, no conformational change is envisaged and hence it is assumed that $Z$ remains the same in both $0 \cdot 01 \mathrm{M}$ and $0 \cdot 1 \mathrm{M}$ salt concentrations.

Then

$\ln \left(k_{0 \cdot 1 \mathrm{M}} / k_{0 \cdot 01 \mathrm{M}}\right)=\left(\Delta E_{a}\right) / R T$

or

$$
\left(\Delta E_{a}\right)=\ln \left(k_{0 \cdot 1 \mathrm{M}} / k_{0 \cdot 01 \mathrm{M}}\right) * R T,
$$

where

$$
\left(\Delta E_{a}\right)=\left[\left(E_{a}\right)\right]_{0 \cdot 01 \mathrm{M}}-\left[\left(E_{a}\right)\right]_{0 \cdot 1 \mathrm{M}} .
$$

\section{Results and discussion}

Identification of the side chains, involved in the epitope interaction, was done by the change in activity of modified derivatives, by the methods described before (Berger et al 1996; Srilatha and Murthy 1996). The results are shown in table 1 . They clearly proved that electrostatic groups like the phenolic, histidinyl and guanidyl are not present at the epitope. Amino group modification resulted in partial loss of activity. Even cyanogen bromide $(\mathrm{CNBr})$ treatment showed marginal role for the methionyl peptide bond/s. The chemically identified core groups, the disulphide bonds and carboxyl groups were inadequate for epitope identification. Data from the dimer $(\alpha \beta)$ modification is much more confusing with increased activity in most modifications, and does not indicate the presence of additional groups at the epitopic region. Consequently identification based on chemical modification was not possible for these two MAbs.

An alternate approach of investigation was based on the fact that the $K_{A}$ is an equilibrium constant; and is a ratio of two independent real time kinetic rate constants, the $k_{+1}$ (forward) and $k_{-1}$ (backward), both of which are inherent constants of the interaction. It is possible to envisage all the three parameters changed by modification. For this reason, kinetics holds definite advantage in splitting 
the $K_{A}$ contributions provided all the three are measured. With the dissociation method (Ashish et al 2002b) it is possible to find $K_{A}, k_{-1}$ and $k_{+1}$ in a single experiment. This advantage has been exploited in mapping the epitopic regions of the MAbs. In analysing the effect of modification, the concentration-independent constant $k_{-1}$ is considered exclusively, as repeated experiments have shown that the precision of measurement of $k_{-1}$ is high $( \pm 15 \%)$. In contrast, the $K_{A}$ and $k_{+1}$ are derived parameters (Ashish et al 2002b). The precision of measurement of these parameters in the experiments done in the laboratory is $\pm 35 \%$. For this reason identification of the residues at epitopes have been based on experimentally determined $k_{-1}$.

Figure 1 shows a representative example of the release of IhCG into solution from immobilized IhCG-MAb complex in the presence of excess unlabelled hCG. The constants $k_{-1}, k_{+1}$ and $K_{A}$ were calculated from such data as described earlier (Srilatha and Murthy 2002; Ashish et al 2002c).

Dissociation rate constants of the hCG/(derivatives)MAb complex are presented in table 2. Data from column 2 , rows $1-4$ showed minimum $k_{-1}$ with $\alpha$-subunit, indicating the epitope-paratope pair to be most stable when hCG is presented as a monomer. Human luteinizing hormone (hLH) that has almost identical structure to hCG (identical $\alpha$-subunit, but has only $1-115$ residues of the $\beta$ subunit, and lacks the $\beta$ CTP $116-145$ of hCG) showed a higher $k_{-1}$, still much lower than hCG. Thus the $\beta$ hCG is blocking the epitopic region in subunit more effectively than the $\beta$ hLH. Acetylation of the amino groups of the hCG did not result in significant change in the $k_{-1}$ indicating little role for the charged amino groups in the epitopeparatope interaction. The rate constant $\left(k_{-1}\right)$ for corresponding tryptic digests (row 5) did not change significantly. However when the trypsin-sensitive bonds are digested in the $\mathrm{hCG} / \mathrm{hLH}$ (rows 6-7), the $k_{-1}$ increased significantly (0.032 to 0.04 in hCG and 0.0198 to 0.0394 in $\mathrm{hLH}$ ), resulting in destabilization of the epitope-paratope pair. In contrast when acetylated hCG (Ac-hCG) was digested with trypsin, the $k_{-1}$ decreased to 0.00947 (column 2: row 8). As $\beta$ CTP is removed in both hCG and Ac-hCG by hydrolysis of the arginine $(\arg )[\beta-\arg 115]$, the difference between the $k_{-1}$ of hCG and Ac-hCG is associated with modification of a lysine (lys) residue within $1-114$ of the $\beta$ hCG. Therefore, a lysine residue is posted near the epitope region whose digestion reduced the $k_{-1}$, but its acetylation did not. This lysine should be present in $\beta$-subunit of hCG/hLH, and should be digestible by trypsin. The only lysine residue so located in $\mathrm{hCG} / \mathrm{hLH}$ is $\beta$-lys 104 . This identification is further substantiated by the observation that chymotryptic digestion of hCG failed to increase the $k_{-1}$ (column 2: rows 10 and 11: $\beta 100-110$ has no chymotrypsin-sensitive bonds). Accordingly, epitope VM11 has in proximity $\beta 100-110$, blocked partly by $\beta$ CTP $(\beta 114-145)$ in native hCG, and is also blocked by the $\beta$-subunit $(\beta 1-114)$ in both hCG and hLH.

In contrast, VM10 showed a different profile for the digested hCG/hLH derivatives (table 2, column 3). Re-

Table 1. Retention of binding activity in hCG derivatives in the SS-SPRIA to immobilized MAb-VM11 and MAb-VM10.

\begin{tabular}{lcccc}
\hline Derivative & $\alpha-\beta$ (VM10) & $\alpha$ (VM10) & $\alpha-\beta$ (VM11) & $\alpha$ (VM11) \\
\hline Control (hCG) & $100^{*}$ & 100 & 100 & 100 \\
TNBS (amino) & 53 & 27 & 30 & 34 \\
TNM (tyr) & 123 & 69 & 348 & 87 \\
Try-digest & 246 & 76 & 333 & 93 \\
Chy-digest & 200 & 115 & 378 & 121 \\
LAP-digest & 85 & 107 & 173 & 107 \\
CPA-digest & 124 & 134 & 138 & 121 \\
HCOOH & 76 & 115 & 188 & 112 \\
CNBr (meth) & 97 & 53 & 169 & 45 \\
CDI (carboxyl) & $<5 \%$ & $<5 \%$ & $<5 \%$ & $<5 \%$ \\
RCMethylated & $<1 \%$ & $<1 \%$ & $<1 \%$ & 100 \\
Acetyl (amino) & 100 & 100 & 100 & 100 \\
DEPC (his) & 100 & 100 & 300 & 85 \\
PG (arg) & & & 120 & \\
\hline
\end{tabular}

Core groups: -COOH/S-S/; -COOH/S-S/.

The proximal groups: -Lys, (Try-sensitive) Meth loop; -Lys, (Try-resistant) Meth loop.

The value points an average of minimum 3 determinations and the error of assay was within $15 \%$.

$* \mathrm{ED}_{50}$ obtained in RIA with normal hCG is considered as $100 \%$. An increased $\mathrm{ED}_{50}$ of the derivative indicates inactivation and decreased $\mathrm{ED}_{50}$ activation. 
moval of the $\beta$ CTP in hCG by trypsin (row 6) and chymotrypsin (row 10) reduced the $k_{-1}$ to $65 \%$, while the $k_{-1}$ for the subunit was least affected (column 3: rows 1,5 and 9). hLH that does not have the $\beta$ CTP also showed a reduction of $35 \%$ in $k_{-1}$ (column 3: rows 1 and 3). Absence of $\beta$ CTP in native hLH did not reduce the $k_{-1}$ to the subunit level, and implied significant blocking of the epitope by the hLH $\beta$-subunit. In addition $k_{-1}$ was not altered by the acetylation (column 3: rows 2 and 4) clearly ruling out the presence of amino groups in interactions with the MAb VM10.

It was observed that even though the $k_{-1}$ for the $\alpha$ hCG was very low (indicating great stability of interaction between $\mathrm{MAb}$ and $\alpha \mathrm{hCG})$, the $K_{A}$ were comparable. Analysis of the $k_{+1}$ showed a surprising observation that despite the reduction in the $k_{-1}, k_{+1}$ increased in the dimer (hCG; tables 2 and 3). The $k_{+1}$ of $\alpha$ hCG was $20 \%$ of the $k_{+1}$ of hCG (table 3, columns 2 and 3: rows 1 and 5). This indicated that the $\beta$-subunit should have some features in it to make this interaction express itself in increased $k_{+1}$. If the interaction is based only on epitope-paratope reaction, it is to be expected that a reduction in $k_{-1}$ should be associated with a reduction in $k_{+1}\left[K_{A}=\left(k_{+1} / k_{-1}\right)\right.$, and $K_{A}$ of subunit and the dimer were comparable]. In this case presence of the $\beta$-subunit made dissociation easy, yet facilitated the binding. This observation suggested that the reactional though explicitly driven by extremely specific epitope-paratope interactions, was neverthless associated with interactions involving other parts of the molecule

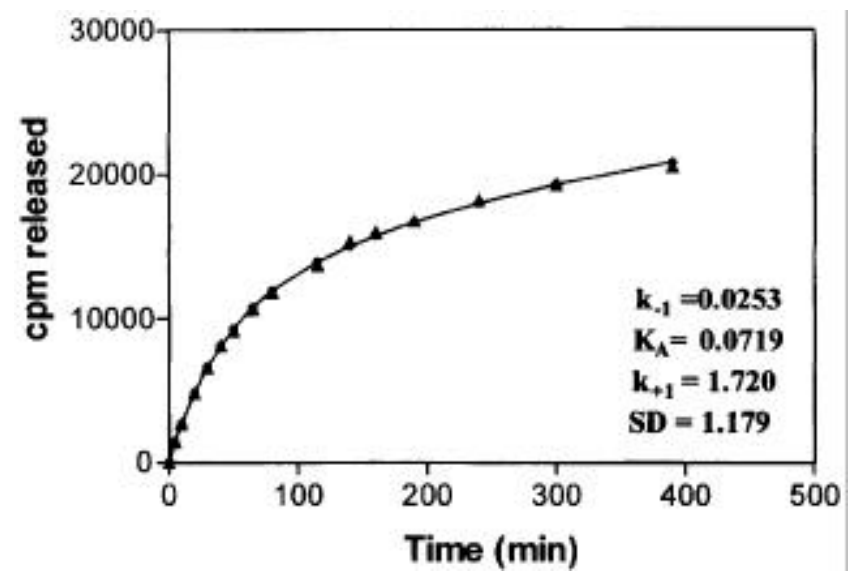

Figure 1. Representative example of the dissociation profile of the IhCG-MAb complex in the presence of excess unlabelled hCG. The curve shows the fit of the data, filled triangles are the experimental points and filled circles are the theoretical points, to a two step reaction (Ashish et al 2002). The parameters obtained experimentally from the above fit were, $k_{-1}$ (dissociation data) and $K_{A}$ (from binding data of hCG-MAb complex formation). $k_{+1}$ was derived from equation $k_{+1}=K_{A} / k_{-1}$ (for details see Ashish et al 2002). SD presented is the average SD of the theoretical and experimental points.
( $\beta$-subunit). Identification of these regions was done based on the $k_{+1}$ obtained for Ac-hCG and its chymotryptic digest (table 3).

Association constants $\left(k_{+1}\right)$ of VM11-hCG reaction (table 3, column 2: rows 5 and 6) showed that despite a large number of chymotryptic cleavages ( 6 numbers) the reduction in the $k_{+1}$ in the $\alpha$ hCG was very marginal. $k_{+1}$ for the dimer is at least 8-6 times more than for the monomer (rows 1 and 3). Obviously, some part of the $\beta$ subunit must be involved in the association. The $\beta$ subunit has two parts, namely the core $\beta$-subunit consisting of $\beta 1-114$ residues and the $\beta$ CTP consisting of 30 residues ( $\beta 115-145$ in hCG) called the tail, as it is not cross-linked with the core region through the disulphide bridges. Therefore, the $\beta$ CTP can be cleaved off the hCG by chymotryptic digestion. Removal of these amino acids in hCG reduced the $k_{+1}$ very significantly both in native hCG (column 2: row 2) and the Ac-hCG (column 2: row 4 ), clearly establishing a role for the $\beta \mathrm{CTP}$ in the association reaction.

In VM10 with $\alpha$ hCG (table 3, column 3: rows 5 and 6 ), chymotryptic digestion did not change $k_{+1}$ and indicated that the epitopic region is left intact by the generation of several additional charges through chymotrypsin cleavage. Increase $(300 \%)$ in the $k_{+1}$ in the dimer $(0.8$ to 2.54: row 1) identified a role for the $\beta$-subunit in the binding reaction. Removal of the $\beta$ CTP by enzyme digestion reduced $k_{+1}$ by $50 \%$ (2.5 to $1 \cdot 375$ : rows 1 and 2 ); clearly proving the importance of the $\beta$ CTP in promoting the association. However removal of the $\beta \mathrm{CTP}$ from Ac-hCG resulted in further decrease of the $k_{+1}$ by $50 \%$ (1.375 to 0.68: rows 2 and 4); suggesting a role for the $\beta$ lys 122 . Yet these results do not provide any evidence to identify the part of the core $\beta$ region responsible

Table 2. $k_{-1}$ values of hCG (derivaties)-MAb complex dissociation profiles $(n=4)$.

\begin{tabular}{lcc}
\hline Protein & $\begin{array}{c}k_{-1}[\mathrm{~mol} / \mathrm{min}] \\
\text { MAb-VM11 }\end{array}$ & $\begin{array}{c}k_{-1}[\mathrm{~mol} / \mathrm{min}] \\
\text { MAb-VM10 }\end{array}$ \\
\hline$\alpha \mathrm{hCG}$ & $0 \cdot 0060 \pm 0 \cdot 00077$ & $0 \cdot 00600 \pm 0 \cdot 00191$ \\
hCG & $0 \cdot 0315 \pm 0 \cdot 03150$ & $0 \cdot 03070 \pm 0 \cdot 00169$ \\
hLH & $0 \cdot 0198 \pm 0 \cdot 00090$ & $0 \cdot 01875 \pm 0 \cdot 00173$ \\
Ac-hCG & $0 \cdot 0368 \pm 0 \cdot 00120$ & $0 \cdot 02808 \pm 0 \cdot 00307$ \\
$\alpha$ hCG-Try & $0 \cdot 00625 \pm 0 \cdot 00035$ & $0 \cdot 00660 \pm 0 \cdot 0002$ \\
hCG-Try & $0 \cdot 04000 \pm 0 \cdot 00340$ & $0 \cdot 01125 \pm 0 \cdot 0011$ \\
hLH-Try & $0 \cdot 03940 \pm 0 \cdot 00859$ & $0 \cdot 01075 \pm 0 \cdot 0019$ \\
Ac-hCG-Try & $0 \cdot 00947 \pm 0 \cdot 00008$ & $0 \cdot 01195 \pm 0 \cdot 0098$ \\
ahCG-Chy & $0 \cdot 0055 \pm 0 \cdot 00067$ & $0 \cdot 00550 \pm 0 \cdot 00072$ \\
hCG-Chy & $0 \cdot 0180 \pm 0 \cdot 00125$ & $0 \cdot 01175 \pm 0 \cdot 00552$ \\
hLH-Chy & $0 \cdot 0154 \pm 0 \cdot 00007$ & $0 \cdot 01060 \pm 0 \cdot 00040$ \\
Ac-hCG-Chy & $0 \cdot 0102 \pm 0 \cdot 00075$ & $0 \cdot 00980 \pm *$ \\
\hline
\end{tabular}

*Represents an average of two determinations. 
for increasing the $k_{+1}$ of the dimer. Since $\beta$ lys 122 was identified, an attempt has been made to find out whether the lysine residue is interacting with the MAb through electrostatic interaction. As electrostatic interaction is easily broken by salt, effect of buffer concentration on the $k_{+1}$ was used to identify the role of $\beta$ lys 122 in the association reaction.

Effect of salt concentration on the $k_{+1}$ for hCG, $\alpha \mathrm{hCG}$, $\mathrm{hLH}$ and Ac-hCG and its chymotryptic digest in $0 \cdot 1 \mathrm{M}$ and $0.01 \mathrm{M}$ buffer concentrations is shown in table 4 . In order to assess the role of the electrostatic interactions between the hCG and MAb, the ratio between the $k_{+1}$ in $0.01 \mathrm{M}$ and $0.1 \mathrm{M}$ buffer is a good index and is independent of the absolute values of the $k_{+1}$. It was found that the $k_{+1}$ was always higher in $0 \cdot 1 \mathrm{M}$ buffer. In VM11 (columns 2-4), the ratio between the $k_{+1}(0 \cdot 1 \mathrm{M} / 0 \cdot 01 \mathrm{M})$ showed that $\alpha \mathrm{hCG}, \mathrm{hLH}$ and Ac-hCG fell into a group where the $k_{+1}$ was increased around $250 \%$. However hCG and Ac-hCG-Chy belonged to a different group with increases of $700 \%$ and $500 \%$ respectively. The increase to $700 \%$ in hCG when compared to $250 \%$ for hLH clearly pointed to the role of the $\beta$ CTP (115-145 of hCG) in the binding. Acetylation of the lys122 (Ac-hCG-Chy) reduced the ratio significantly $(500 \%$ from $700 \%$ column 4 : rows 2 and 6). Hence part of the increase in the ratio in hCG

Table 3. Association constants $\left(k_{+1}\right)$ of the reaction between IhCG derivatives and immobilized MAb derived from the dissociation data.

\begin{tabular}{|c|c|c|}
\hline hCG derivative & $\begin{array}{c}\text { MAb-VM11 } \\
\left(k_{+1}\right)\end{array}$ & $\begin{array}{c}\text { MAb-VM10 } \\
\left(k_{+1}\right)\end{array}$ \\
\hline $\mathrm{hCG}$ & $5 \cdot 70 \pm 0 \cdot 365$ & $2 \cdot 540 \pm 0 \cdot 465$ \\
\hline hCG-Chy & $2 \cdot 01 \pm 0.299$ & $1 \cdot 375 \pm 0 \cdot 262$ \\
\hline Ac-hCG & $6 \cdot 97 \pm 1 \cdot 350$ & $3 \cdot 500 \pm 0.495$ \\
\hline Ac-hCG-Chy & $1 \cdot 61 \pm 0.062$ & $0 \cdot 680 \pm 0.050$ \\
\hline$\alpha \mathrm{hCG}$ & $0 \cdot 66 \pm 0.052$ & $0.820 \pm 0.401$ \\
\hline$\alpha$ hCG-Chy & $0 \cdot 42 \pm 0 \cdot 032$ & $0 \cdot 740 \pm 0 \cdot 057$ \\
\hline
\end{tabular}

can be attributed to the lys 122 . The other part is also contributed by charges, but the nature of the charge involved in this interaction could be either arg133 or a negativelycharged acidic group. We have no evidence to rule out carboxyl group involvement in this interaction. A sterical blocking of the epitope region by $\arg 133$ is also not ruled out. The results for VM10 showed a different profile (table 4: columns 5-7). While the ratio of $k_{+1}$ remained similar in $\alpha \mathrm{hCG}, \mathrm{Ac}-\mathrm{hCG}$ and Ac-hCG-Chy digest (rows 3,5 and 6), it differed in hLH and hCG (500\%). The involvement of the core $\beta$-subunit in the binding through electrostatic interaction is thus confirmed.

Figure 2 represents the identification/localization of the epitope VM11 based on results from chemical modification, real time kinetics, and the X-ray structure. The identification of the $\beta 100-110$ has been obtained based on the data from enzyme digests. Other regions were identified from the topography of the $\beta \mathrm{CTP}$ in hCG (Venkatesh et al 1999). Chemical modification data identified the presence of a lysine distant to epitopic region, and is probably the $\alpha$ lys63. CNBr breaks the loops 60 82 , and disturbing the region 54-59 and hence resulting in partial loss of activity. The loss of activity by carboxyl group modification is because of the presence of glutamic acids (glu) [ $\alpha$ glu9] at the epitope, and seems to be crucial in the interaction with the MAb. Changes in $k_{-1}$ by enzyme digestion identified $\beta 100-110$ as a part of the epitope. Thus the $\beta$ CTP blocks the epitope region significantly in $\mathrm{hCG}$, corroborating the conclusions drawn from the kinetics (table 4). The core region of $\beta$-subunit is behind the epitope region and does not block the epitope. The role of $\beta$ lys122 is very clearly marked as the amino acid blocking the epitope region, sitting directly on the $\beta 100-110$ loop. The core residues identified are $\alpha 5-10$ and $\alpha 54-62$, the later being blocked by $\beta 100-110$ in native hCG and $\mathrm{hLH}$. The effective epitope in hCG and hLH will be $\alpha 5-$ 10 (figure 2), while in the $\alpha \mathrm{hCG}$ it is $(\alpha 5-10+\alpha 55-62)$, connected through the 10-60 disulphide bridge.

Figure 3 represents the epitope for VM10. The region interacting with the paratope is most likely $\alpha 4-14$. It is

Table 4. Effect of salt concentration on the $k_{+1}$ in MAb-hCG interaction.

\begin{tabular}{|c|c|c|c|c|c|c|}
\hline \multirow{2}{*}{$\frac{\text { MAb }}{\text { Salt conc. }}$} & \multicolumn{2}{|c|}{ VM 11} & \multirow[b]{2}{*}{ Activity $(\%) *$} & \multicolumn{2}{|c|}{ VM 10} & \multirow[b]{2}{*}{ Activity $(\%)^{*}$} \\
\hline & $0 \cdot 1 \mathrm{M}$ & $0 \cdot 01 \mathrm{M}$ & & $0 \cdot 1 \mathrm{M}$ & $0 \cdot 01 \mathrm{M}$ & \\
\hline HCG & $5 \cdot 7$ & $0 \cdot 810$ & 700 & $2 \cdot 500 \pm 0 \cdot 465$ & $0 \cdot 510 \pm 0 \cdot 154$ & 500 \\
\hline $\mathrm{AhCG}$ & $0 \cdot 660 \pm 0 \cdot 0516$ & $0 \cdot 210 \pm 0 \cdot 0259$ & 300 & $0 \cdot 820 \pm 0 \cdot 401$ & $0 \cdot 590 \pm 0.228$ & 140 \\
\hline HLH & $1.595 \pm 0.0626$ & $0 \cdot 652 \pm 0.0626$ & 250 & $1.025 \pm 0.423$ & $0.243 \pm 0.0289$ & 430 \\
\hline Ac-hCG & $6 \cdot 970 \pm 1 \cdot 3500$ & $2 \cdot 950 \pm 0 \cdot 3290$ & 240 & $3 \cdot 500 \pm 0 \cdot 495$ & $1 \cdot 830 \pm 0 \cdot 147$ & 200 \\
\hline Ac-hCG-Chy & $1 \cdot 610 \pm 0.0622$ & $0 \cdot 275 \pm 0 \cdot 3290$ & 500 & $0 \cdot 680 \pm 0$ & $0 \cdot 445 \pm 0$ & 150 \\
\hline
\end{tabular}

*Percent activity is represented using $0.01 \mathrm{M}$ value as $100 \%$. 

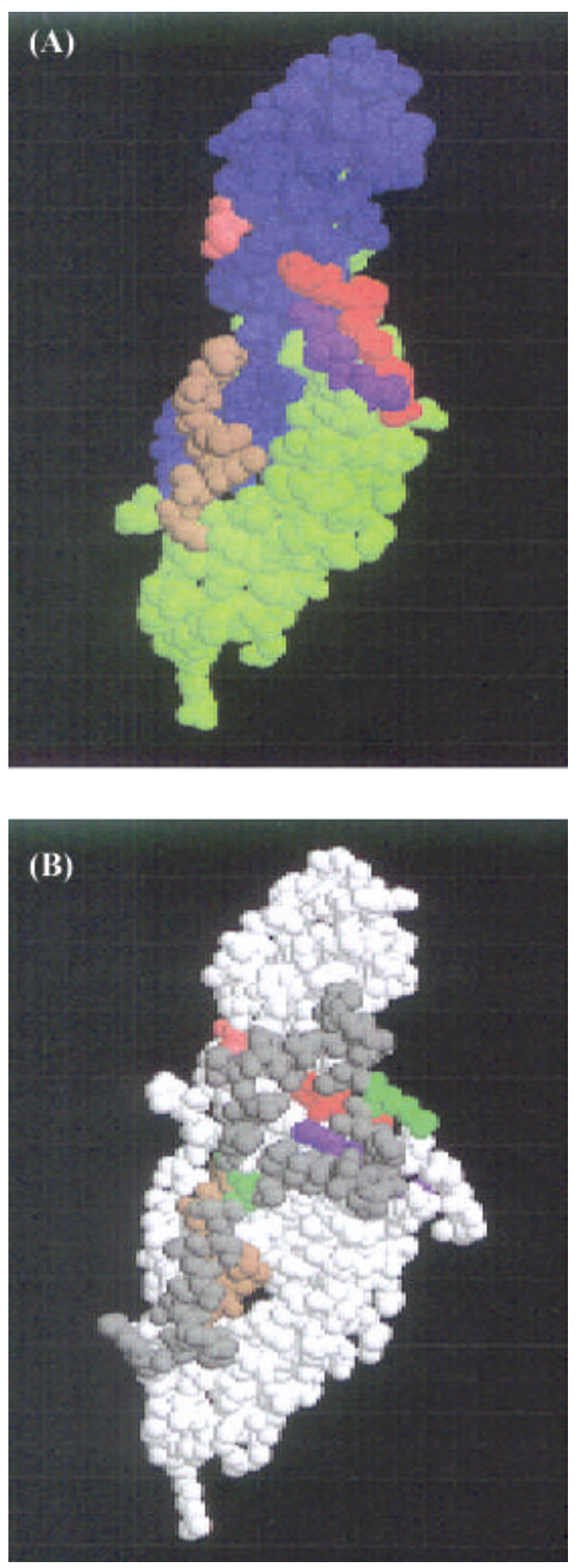

Figure 2. VM 11 epitope in hLH (A) and in hCG (B). (A) Subunits in blue and green. The red colour present $\alpha 6-10$ residues identifying the exposed epitope region adjascent to $\beta 3-6$, region $\alpha 56-62$ (epitope region) being blocked by $\beta 100-110$. (B) The identification of the epitope in the hCG, which has the $\beta C T P$ (shown in grey) covering part of the epitopic region. Other colour codes are as follows. Brown $\beta 100-110$; purple $\beta 3-6$; red $\alpha 6-10$; pink $\alpha$ lys 63 ; green $\beta$ lys122 and $\arg 133$; and grey $\beta 115-145$. seen from figure $3 \mathrm{~B}$ that the region of the $\alpha \mathrm{hCG}$ that is blocked by $\beta C T P$ is the region $\alpha 4-14$. It has one aspagine (Asn) [ $\alpha 6]$ and two glutamic acid residues ( $\alpha 9$ and 14), whose modification inactivated the subunit. Except for these, the region does not have residues amenable for modification explaining the stability of the binding activity to other modifications. Removal of the $\beta \mathrm{CTP}$ (like $\mathrm{hLH}$ ) completely exposed the epitope (figure $3 \mathrm{~A}$ ) and reduced the $k_{-1}$ (increased the stability). The proximity of the region $\beta 3-6$ to the region $\alpha 4-9$ indicates partial blocking of the epitopic region by $\beta 3-6$. Accordingly the epitope region VM10 consists of $\alpha 4-14$ partially blocked by $\beta 3-6$. The changes in the $k_{+1}$ among the $\alpha \mathrm{hCG}, \mathrm{hLH}$ and hCG can be attributed to the olys63 and $\beta$ arg133, acting as incidental interacting regions (IIR). In $\mathrm{hLH}$ alys63 is not blocked by $\beta$ CTP and hence acts as an IIR away from the epitopic region. However in the native hCG the $\alpha$ lys63 is sterically blocked by the $\beta$ CTP. Instead, $\beta \arg 133$ acts as an IIR located close to the main epitope $\alpha 9-14$, blocking the more important binding site (as indicated by $k_{-1}$ much higher than that of hLH).

The role of the lys122 in the specific (epitope-paratope) interaction of hCG with MAb-VM11 was analysed by studying the effect of acetylation on the $k_{-1}$ of the interaction. Table 5 presents the $k_{-1}$ values for hCG, AchCG and their enzyme digests at $0 \cdot 01 \mathrm{M}$ and $0 \cdot 1 \mathrm{M}$ buffer. Changes in the rate constant of hCG/analogues in $0 \cdot 1 \mathrm{M}$ salt indicated (as already described) the role for the $\beta \mathrm{CTP}$ in blocking the epitope. Acetylation of hCG did not increase the $k_{-1}$ significantly. A comparison in the extent of reduction of $k_{-1}$ relative to Ac-hCG (table 5: columns 2 and 4 ) indicated that all analogues/derivatives showed a reduction in activity to the same extent (40\% columns 3 and 5: rows 4 and 5) except Ac-hCG (row 3). In Ac-hCG, $k_{-1}$ increased by $225 \%$ (column 5: row 2). Hydrolysis (by try and chy) of Ac-hCG reverted the pattern of change, establishing that lysine residues (12 numbers) of the subunits $(\alpha 1-92$ and $\beta 1-115)$ have no role in altering the $k_{-1}$. Even though many additional charges are introduced into the molecule during enzyme digestion of Ac-hCG, increase in the total number of charges (both positive and negative), as a whole did not alter the $k_{-1}$ pattern. Interestingly change of a single charge in $\beta$ CTP in Ac-hCG changes the pattern drastically, indicating the involvement of lysine/s as a charged residue at the epitopic region acting through the electrostatic interaction. In contrast to the Ac-hCG, its digests did not destabilize the complex (rate constant did not increase) proving that the lysine we are interested is located in the 114-145 region of the $\beta$ CTP. Hence the lysine residue present at the epitope region should be $\beta$ lys 122 . The X-ray structure identifies this residue to be blocking the epitope region and yet interacts with some part of the MAb through 

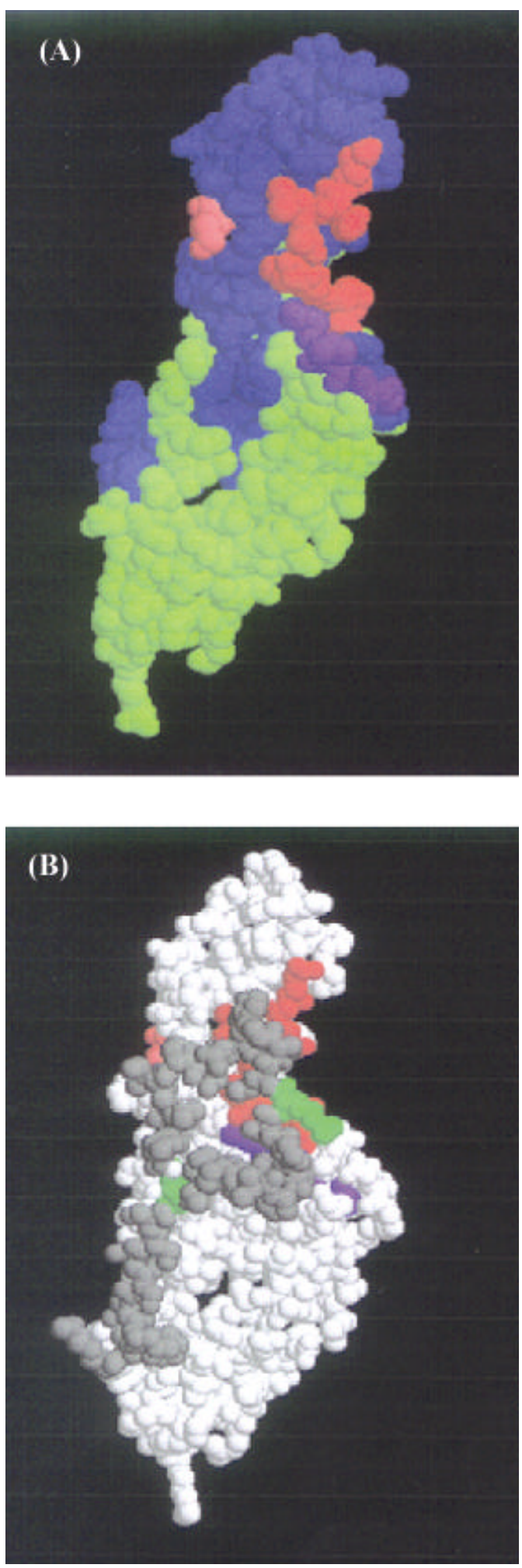

Figure 3. VM 10 epitope in hLH (A) and in hCG (B). (A) Subunits in blue and green and the red colour presents $\alpha 4-14$ which constitutes the epitope region of VM 10 in hLH. (B) The epitope in hCG, partially covered by $\beta \mathrm{CTP}(\beta 115-145$ in grey). Other colour codes are as shown in figure $2 \mathrm{~B}$. electrostatic force. Consequently $\beta$ lys 122 becomes a region of interaction between VM11 and hCG, masking the $\alpha$ residues, and yet increasing the $k_{+1}$, and becomes an incidental epitope. It is incidental because the native epitope is blocked by this lysine, but in this process provides a charged residue for possible electrostatic interaction, which can incidentally occur with a negatively charged group of the MAb, placed spatially in favourable position.

The results of VM10 (table 4: column 7) showed the involvement of the core $\beta$-subunit in the binding through electrostatic interaction. The amino group could be placed in the core $\beta$-subunit or in the $\alpha \mathrm{hCG}$, as both hLH and hCG showed the increase (table 4: column 7: rows 2 and 4). The only lysine residue that fits into the region was found to be $\alpha$ lys 63 , and none in the $\beta$. Thus it is likely that the $\alpha$ lys63 (located away from the epitopic region) is the residue involved in the association reaction of hLH with the MAb VM10 (away from the epitopic region). However, lys63 is blocked in the hCG by the CTP (figure 3). Therefore, the electrostatic interaction seen in the hCG-MAb (VM10) is through other charged residue other than alys63. This residue is most likely $\beta \arg 133$. It is not blocking the epitope significantly, can become a point of interaction with a negatively charged residue of the MAb VM10, and accounts for the experimental observations (table 4: column 7).

Comparing results of epitope mapping obtained from the above methods and those obtained exclusively from chemical modification studies would be interesting. Chemical modification studies would have identified VM10 epitope region as consisting of the disulphide/s, carboxyl group/s, a loop region containing a methionine (meth) residue, and presence of a lysine away from the epitope region, and absence of regions containing histidine (his), arginine, tyrosine (tyr). The epitope should also be away from easily digestible lysine and arginine residues. This method of analysis would be by elimination, than by positive identification. The region that satisfies all the above criteria would be the region around $\alpha 7-10$, cys10-60 and lys63, cys32, cys31. The loss of activity on tryptic digestion in VM10 would identify the region 3259 as a loop region giving stability to the epitope (lys44, lys45, $\arg 42$, lys51 are digestable). Thus the region identified would be the core region 7-10, 60-63 with the disulphide bond 10-32 stabilizing the epitope. Likewise the region identified for the VM11 would be cys10, cys60, cys59 and cys32. Accordingly, even in the absence of Xray structure and kinetics, the identification of the core region would not have been grossly wrong. In fact based on the results coming from chemical modification studies, we infer that the peptide which could be a synthetic substitute for epitope VM10, would be the one shown below where $\mathrm{X}$ represents an amino acid which needs to be in- 
Table 5. Effect of acetylation on the salt dependent $k_{-1}$ in the MAb-hCG interaction (role of the lys 122 in the core region interaction in VM11).

\begin{tabular}{lcccc}
\hline Derivative & $\begin{array}{c}0 \cdot 1 \mathrm{M} \\
k_{-1}(\mathrm{~mol} / \mathrm{min})\end{array}$ & $\begin{array}{c}0 \cdot 1 \mathrm{M} \\
\text { Activity }(\%)\end{array}$ & $\begin{array}{c}0 \cdot 01 \mathrm{M} \\
k_{-1}(\mathrm{~mol} / \mathrm{min})\end{array}$ & $\begin{array}{c}0 \cdot 01 \mathrm{M} \\
\text { Activity (\%) }\end{array}$ \\
\hline hCG & 0.0315 & 100 & $0 \cdot 0220$ & 100 \\
Ac-hCG & 0.0368 & 117 & $0 \cdot 0495$ & 225 \\
Ac-hCG-Try & 0.0095 & 30 & $0 \cdot 0060$ & 27 \\
Ac-hCG-Chy & 0.0102 & 32 & $0 \cdot 0086$ & 39 \\
\hline
\end{tabular}

corporated between cys32 and cys59 through peptide bonds. The nature of the amino acid cannot be identified right now.

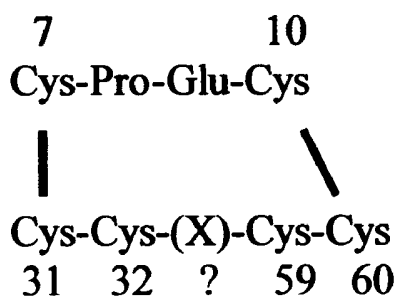

However, X-ray structure consolidates the epitope analysis because of positive identification of the residues. These data also indicate that the negative identification approach is complementary and not contradictory to the kinetic approach as done here. Identification of the epitopes as shown in figures 2 and 3 conform to all the experimental results. The observation that modifications failed to identify the epitope can be easily attributed to the fact that the regions of the epitopes do not have side chain residues amenable for modification. In consequence the interaction is more hydrophobic in nature than with other MAbs reported earlier.

One of the surprising observations we came across during these studies was that low-salt concentration changed the $k_{+1}$ profoundly compared to $k_{-1}$ (tables 4 and $5)$. The change in the concentration of salt is low enough to influence the electrostatic interaction but not the hydrophobic interactions. Salt at this concentration (0.01$0 \cdot 1 \mathrm{M})$ is unlikely to alter the three-dimensional structure of the molecules. This is justified by the observation that even gross modifications in hydrolysed hCG with increased number of charges failed to prevent the binding establishing the intact nature of the epitope after digestion (Venkatesh and Murthy 1997a). The hydrophobic interactions are likely to be altered only at very high salt concentrations well above $1 \mathrm{M}$ ammonium sulphate, used in hydrophobic interaction chromatography (Amersham Pharmacia biotech manual on Hydrophobic interaction Chromotography, p. 17). Therefore, we believe that the interaction that resulted in increased $k_{+1}$ should be away from the epitope-paratope site, and should form an IIR. Such interaction is likely to involve the side chain charged groups of lys, arg, his, or carboxyl groups of acidic amino acids and other side chains of hydrophobic amino acids. The role of the $\varepsilon$-amino groups of lysine in this interaction can be inferred by the $k_{+1}$ for the Ac-hCG. Using the $\mathrm{X}$-ray structure the possible amino group involved in such interaction has also been identified. However identification of the involvement of other amino acids (arg, his, asp and glu) in such interactions are still speculative. An extension of this incidental interaction near the epitope-paratope pair results in an incidental epitope, as it has happened in the case of the epitope VM10. The incidental epitope here is the $\beta$ lys 122 . These incidental interactions (whether at epitope or away from the epitope) occur only in low salt and not associated with conformational changes of either the hCG or its MAb. They affect mostly the $k_{+1}$ and do not affect the $k_{-1}$ significantly. The IIR can be expected to be a region where salt bridge type of interaction is occurring between the charged groups of the reactants with distances large enough to allow the water molecules to be present between them. Diagrammatic representation of incidental interaction is shown in figure 4 . In the presence of salt the positions of the reacting groups are not altered. But the presence of salt between these groups reduces the contribution of the energy to the total interaction. Since the $k_{-1}$ is not altered this change of energy was attributed to incidental region and not to epitope-paratope pair. Such incidental interactions in our study is the interaction between $\alpha$ lys63 (in $\mathrm{hLH}$ ) and $\beta \arg 133$ (in hCG) with a charged group on the MAb VM11. Thus $\beta \arg 133$, which is blocking the region $\alpha 27-14$ acts as an incidental blocking region, increasing the $k_{+1}$ four-fold 0.42 to 1.61 (see table 3: column 2).

The incidental regions of interactions identified in this study have been $\alpha$ lys63, $\beta$ lys 104, $\beta$ lys 122 and $\beta \arg 133$. These incidental regions are not located at the epitope region, and yet they interact with the MAbs through the electrostatic charges on the side chain. They are able to change the association rate constant significantly from bringing down the activation energy. $\beta$ lys122 and $\beta$ lys 104 act as incidental regions located close to the epitope affecting the $k_{-1}$ significantly in VM11. The exact 
contribution of the incidental regions to reduce the activation energy can be obtained only in those instances where $k_{-1}$ is minimally altered. In other cases it would be qualitatively identified, but cannot be quantified. Such incidental interacting regions may be present in many protein-protein complexes, and their identification can be done using the kinetic approach.

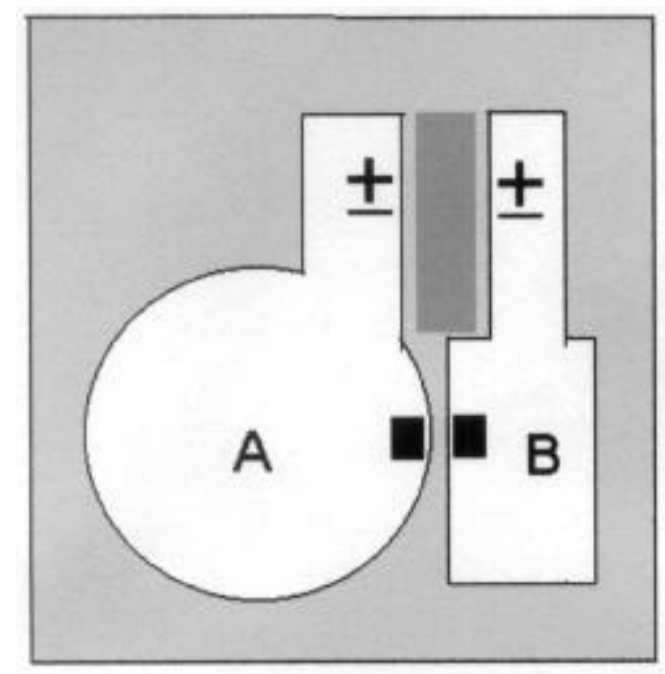

Figure 4. Diagrammatic representation of the IIR. ' \pm ' represents the charges (or charge clusters) on the MAb and hCG. A and $\mathrm{B}$ represent hCG and MAb. Filled (black) rectangles indicate the epitope and paratope pair. ' \pm ' in both molecules present charged residues in hCG and MAb separated by sufficient distance to prevent any charge interaction at high salt concentration, but small enough to permit interaction at low concentration. The grey rectangle presents the area of proximity of the interacting regions with full access to water and salt. If the charges are the same in both the paratope and epitope it results in repulsion at low salt concentration, and hence reduced rate of reaction (ex hCG-MAb). If they are different, reaction will be faster in the absence of salt.
The nature of interaction of incidental regions with $\mathrm{MAb}$ was assessed by a direct experiment involving the measurement of the cross reactivity of $\alpha \mathrm{hCG}, \mathrm{CNBr}-$ digested subunit and the tryptic and chymotryptic digests of the later. Cross reactivity of the above derivatives are shown in table 6 . The relative affinity constant $\left(K_{A}\right.$ determined by the percent cross reactivity) is altered when a significant part of the peptide chain comes off. The change is marginal between $\alpha \mathrm{hCG}$ and $\mathrm{CNBr}-\alpha \mathrm{hCG}$ $(<40 \%)$ where two peptide bonds are cleaved breaking two important loop regions (32-59 and 60-82), but without release of any amino acid from the protein (figure 5B). In contrast the binding of the try/chy digest of $\mathrm{CNBr}$ hCG resulted in greater decrease of cross-reactivity $(87 \%$ and $94 \%$ for VM11 and $94 \%$ and $98.5 \%$ for VM10, table $6)$. Tryptic and chymotryptic digestion results in loss of 28 and 27 amino acid residues respectively (figure 5C,D). This certainly prove that some amino acids in the loop regions of 35-47 and 60-82 are involved in the binding reaction. These data clearly provide an evidence for the important role played by apparently non-interacting regions of the primary structure in enhancing the binding of the MAb to hCG and its derivatives. The reason why the chymotryptic digestion causes greater loss of activity can be envisaged from the primary structure, where the $\alpha$ phe20 21 bond is a susceptible site and its cleavage (by chymotrypsin) could results in the breaking of the loop region 10-28. The presence of IIR's is further supported by the observation that salt dependent changes in the $k_{-1}$ were marginal $(<50 \%)$, as against larger changes in $k_{+1}$ (>300\%) (table 7).

Quantitative measurement of changes in the rate constants offer an opportunity to quantify the changes in activation energy brought on by such interactions in situations where the $k_{+1}$ is altered but not the $k_{-1}$. In our study this is the case with the interaction of the MAb VM11 with Ac-hCG-chy (table 7: columns 6 and 7: rows

Table 6. Cross reactivity of derivatives of $\alpha \mathrm{hCG}$ with MAbs**.

\begin{tabular}{lcc}
\hline Complex used in dissociation & $\begin{array}{c}\text { Sp.binding in } \\
\text { SS-SPRIA }(\%)\end{array}$ & $\begin{array}{c}\text { Number of amino } \\
\text { acids lost }\end{array}$ \\
\hline VM 11-hCG* & 100 & - \\
VM 11- $\alpha$ hCG & 85 & 0 \\
VM 11- $\alpha$ hCG-CNBr & 50 & 0 \\
VM 11- $\alpha$ hCG-CNBr-Try-digest & 13 & 28 \\
VM 11- $\alpha$ hCG-CNBr-Chy-digest & 6 & 27 \\
VM 10-hCG* & 100 & - \\
VM 10- $\alpha$ hCG & 90 & 0 \\
VM 10- $\alpha$ hCG-CNBr & 54 & 0 \\
VM 10- $\alpha$ hCG-CNBr-Try-digest & 4 & 28 \\
VM 10- $\alpha$ hCG-CNBr-Chy-digest & $1 \cdot 5$ & 27 \\
\hline
\end{tabular}

* Specific binding of the native hormone is considered $100 \%$.

**The values present average of at least 2 determinations. 
4 and 5). The change in the $\left(\Delta E_{a}\right)$ was $1.095 \mathrm{kcal}$, yet the changes in the rate constant $\left(k_{+1}\right)$ was almost 5-fold (columns 3, 4: row $5\{1 \cdot 61 / .275\})$. The normal activation energy is around $20 \mathrm{kcal}$ (Tamil Selvi et al 2002; Ashish et al 2002c) and hence the change in the activation energy was only $5 \%$, yet the change in the rate constant was $500 \%$. Obviously, kinetics provides an opportunity to estimate small changes in the activation energy by measuring the changes in $k_{+1}$. The assumption made is that identical conformation is retained by MAb, hCG and the MAb-hCG complex at both salt concentrations ( $0.1 \mathrm{M}$ and $0.01 \mathrm{M})$. Then $\left(\Delta E_{a}\right)$ quantifies the contribution coming from the incidental interactions (column 5). The data also brings out the extent of contribution to the activation energy through the $\beta C$ TP. Removal of $\beta C T P$ reduces the $\left(\Delta E_{A}\right)$ in VM11 by $\sim 600$ cals (column 5: \{rows $4-5$; 1095-530 cal $\}$ ). Similarly the contribution (ionic strength dependent) of $\beta \mathrm{CTP}$ is insignificant in VM10-MAb (<200 cals) (column 5: \{row 6-row 1: 670-503 cal \}).

The reason for the change in the $k_{+1}$ on loss of the noninteracting region can be obtained by simple calculations on the extent of the decrease in activation energy required to bring down the rate of association. A reduction of activation energy by 5\% [1000 cal, assuming the activation energy to be $20 \mathrm{kcal}$ (Tamil Selvi et al 2002)] changes the $k_{+1}$ five-fold, and a change of 2000 cal (a change of $10 \%$ ) by as much as 30 -fold. This assumes that the $k_{-1}$ is not much altered during the modifications, which are justified by the data obtained (table 6). The investigations have shown that the change in the activation energy can be as much as $1000 \mathrm{cal} /$ pair for chargecharge interaction (table 6). Considering that the binding reaction can have a hydrophobic component it is easy to envisage significant changes in the activation energy through incidental interactions away from the active site. Thus the data signifies the importance of the apparently non-specific non-interacting regions in driving the association reaction to favour complex formation. This may explain the importance of $\alpha \mathrm{hCG}$ in eliciting biological response only when associated with the $\beta$-subunit. Likewise the failure of the $\beta$-subunit to bind significantly to the receptor in the absence of the $\alpha$ hCG can be explained on the basis of IIR. In this case IIR can be expected to be in the $\alpha$ hCG facilitating the binding of the dimer to the region of the receptor involved in eliciting biological response. The importance of the specificity of the interac-
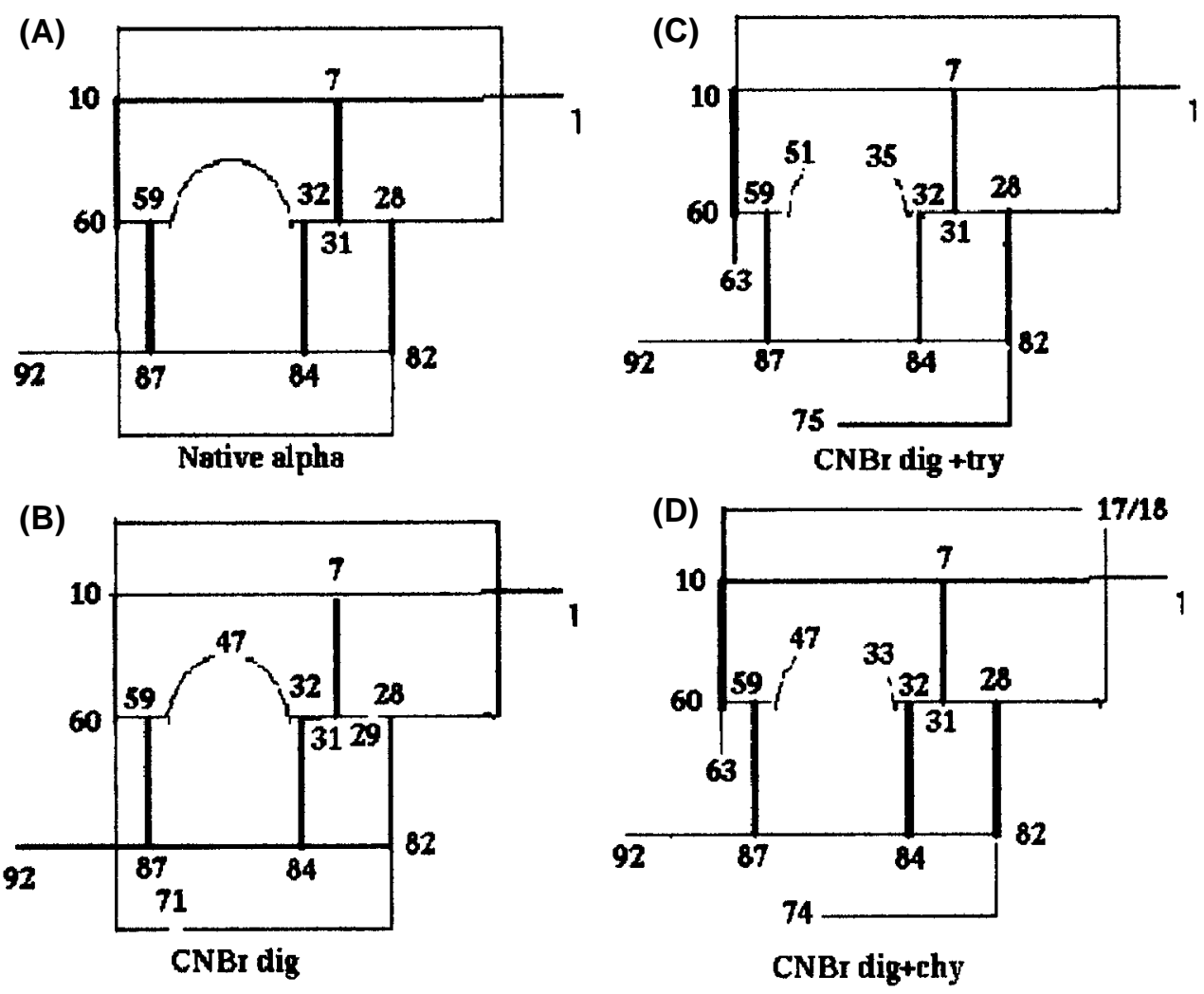

Figure 5. Diagrammatic representation of the hydrolysis of the $\alpha$ hCG (A), $\alpha$ hCG-CNBr (B), ahCG.CNBr-Try digest (C) and $\alpha$ hCG.CNBr-Chy digest (D). (B) has two bonds broken at met 29 and met 71 with no loss of amino acids. (C) has several tryptic cleavages resulting in the release of amino acids 35-51 and 64-75. (D) has lost residues 33-47, 89-92 and a cleavage at phe 19. 
Table 7. Changes in the ionic strength dependent activation energy $\left(\Delta E_{a}\right)$ in hCG-MAb interaction.

\begin{tabular}{|c|c|c|c|c|c|c|}
\hline \multirow[b]{2}{*}{ MAb } & \multirow[b]{2}{*}{ Ligand } & \multicolumn{2}{|c|}{$k_{+1}\left(\times 10^{6} \mathrm{M}^{-1} \min ^{-1}\right)$} & \multirow{2}{*}{$\begin{array}{l}\mathrm{cal} / \mathrm{mol} \\
\left(\Delta E_{a}\right)^{* *}\end{array}$} & \multicolumn{2}{|c|}{$k_{-1}\left(\min ^{-1}\right)$} \\
\hline & & $0 \cdot 1 \mathrm{M}$ & $0 \cdot 01 \mathrm{M}$ & & $0 \cdot 1 \mathrm{M}$ & $0.01 \mathrm{M}$ \\
\hline VM 10 & hCG-Chy & $1 \cdot 375$ & $0 \cdot 610$ & 503 & $0 \cdot 01175$ & 0.01176 \\
\hline VM 10 & Ac-hCG & $3 \cdot 505$ & 1.830 & 403 & 0.02808 & 0.03237 \\
\hline VM 10 & Ac-hCG-Try & $1 \cdot 310$ & $0 \cdot 510$ & 578 & 0.01195 & $0 \cdot 01140$ \\
\hline VM 11 & hCG-Chy & $2 \cdot 000$ & $0 \cdot 850$ & 530 & $0 \cdot 01800$ & 0.02000 \\
\hline VM 11 & Ac-hCG-Chy & $1 \cdot 610$ & $0 \cdot 275$ & 1095 & $0 \cdot 01020$ & 0.00870 \\
\hline VM 10 & hCG & $2 \cdot 540$ & $0 \cdot 510$ & $670^{*}$ & 0.0307 & $0 \cdot 0173$ \\
\hline VM 11 & hCG & $5 \cdot 700$ & $0 \cdot 800$ & $1178 *$ & $0 \cdot 03150$ & 0.0220 \\
\hline
\end{tabular}

*These values have been calculated assuming minimum interference from the differences in the dissociation constants. Hence, they are approximate.

$* *$ Represents the increase/decrease in activation energy affected by salt in the association reaction $\left(E_{0 \cdot 01}-E_{0 \cdot 1}\right)$.

tion lies in the site defined as the binding region, but the incidental interactions may determine the binding affinity $K_{A}$ and hence subsequent expression of biological activity. This is justified by the observation that homodimer $\beta-\beta$ (Lobel et al 1999) and linked $\beta-\alpha$ dimer (Jackson et al 1999) have considerable bioactivity in vitro. The disposition of the charges in the loop regions is similar in the two subunits and hence it is possible to envisage the clusters of the charges/hydrophobic residues of one $\beta$ subunit simulating the $\alpha$-subunit in the expression of bioactivity in the $\beta-\beta$ dimer. The type of the interaction may not necessarily be electrostatic in nature, as the $k_{-1}$ is also altered on the removal of the loop regions (figure 4 and table 6) indicating their involvement in the hydrophobic and other interactions also. Consequently, IIRs become important in both the binding and the expression of biological activity. The role of electrostatic interaction in the plasticity of proteins was indicated earlier by theoretical considerations (Kumar and Nussinor 2002; Mohan et al 2002, 2003; Li et al 2003; Sinha et al 2002; Sinha and Smith Gill 2002). In this paper we have practically demonstrated and identified such incidental regions, and to our knowledge this is the first demonstration of delineating the IIR by simple chemical approaches. Thus real time kinetic methods provide a tool by which one can identify and investigate quantitatively their significance in chemical and biological processes.

In conclusion, we have described a real time kinetic method for identification of epitopes refractory to chemical modifications in hCG. Epitope mapping data coupled with the kinetic data has identified the importance of non-epitope regions as IIR in hCG-MAb interaction. It is demonstrated by simple binding data that though disulphides are important for the epitope structure the $K_{A}$ of interaction is influenced substantially by the IIRs, but they have no role in determining the specificity of the interaction.

\section{Acknowledgements}

We thank Department of Science and Technology, New Delhi for financial support.

\section{References}

Ashish B, Venkatesh N and Murthy G S 2002a Structure function analysis: Lessons from human chorionic gonadotropin; Indian J. Exp. Biol. 40 434-447

Ashish B, Srilatha N S and Murthy G S 2002b Real time kinetic analysis of hCG-monoclonal antibody interaction using radiolabelled hCG probe: Presence of two forms of Ag-MAb complex as revealed by solid phase dissociation studies; Biochim. Biophys. Acta 1569 21-30

Ashish B, Tamil Selvi P and Murthy G S 2002c Thermodynamics of hCG-monoclonal antibody interaction: an analysis of real time kinetics data obtained using radiolabelled hCG probe; Biochim. Biophys. Acta 1572 31-36

Berger P, Bidart J M, Delves P S, Dirnhofer S, Hoermann R, Isaacs $\mathrm{N}$, Jackson $\mathrm{A}$, Klonisch $\mathrm{T}$, Lapthorn $\mathrm{A} \mathrm{J}$, Lund $\mathrm{T}$, Mann K, Roitt I, Schwarz S and Wick G 1996 Immunochemical mapping of gonadotropins; Mol. Cell Endocrinol. $12333-43$

Fraker P J and Speck J C 1978 Protein and cell membrane iodination with sparingly soluble chloramide 1, 3, 4, 6-tetrachloro3, 6-diphenylglycouril; Biochem. Biophys. Res. Commun. 80 $849-857$

Jackson A M, Berger P, Pixley M, Klein C, Hsueh A J and Biome I 1999 The biological action of choriogonadotropin is not dependent on the complete native quaternary interactions between the subunits; Mol. Endocrinol. 13 2175-2188

Kumar S and Nussinov R 2002 Relationship between ion pair geometries and electrostatic strengths in proteins; Biophy. $J$. 83 1595-1612

Lapthorn A J, Harris D C, Littlejohn A, Lustbader J W, Canfield R E, Machin K J, Morgan F J and Isaacs N W 1994 Crystal Structure of human chorionic gonadotropin; Nature (London) 369 455-461

Li Y, Li H, Yang F, Smith-Gill S J and Mariuzza R A 2003 Xray snap shots of the maturation of the antibody response to a protein antigen; Nat. Struct. Biol. $10482-488$ 
Lobel L, Pollak S, Wang S, Chaney M and Lustbader J W 1999 Expression and Characterization of recombinant $\beta$-subunit hCG homodimer; Endocrine 10 261-270

Matzuk M M, Hsueh A J W, LaPolt P, Tsafriri A, Keene J L and Biome I 1990 The biological role of the carboxy-terminal extension of human chorionic gonadotropin; Endocrinology $126376-383$

Mohan S, Sinha N and Smith-Gill S J 2002 Differences in electrostatic properties at antibody-antigen binding sites: implications for specificity and cross-reactivity; Biophys. J. 83 2946-2968

Mohan S, Sinha N and Smith-Gill S J 2003 Modeling the binding sites of anti-hen egg white lysozyme antibodies Hy-HEL8 and Hy-HEL-26: an insight into the molecular basis of cross-reactivity and specificity; Biophys. J. 85 32213236

Murthy G S 1996 Real time kinetic analysis of antigen-antibody interaction using solid phase binding: Transformation of hCG- monoclonal antibody complex; Curr. Sci. 71 981-988

Murthy G S and Srilatha N S 1996 Mapping of assembled epitopes with microgram quantities of antigen: Identification of an epitope at the receptor binding region of hFSH; Curr. Sci. 70 1019-1022

Murthy G S and Venkatesh N 1996 Determination of kinetics of epitope paratope interaction based on solid phase binding: an inexpensive alternative to biospecific interaction analysis; J. Biosci. 21 641-651

Purohit S, Shao K, Balasubramanian S V and Bahl O P 1997 Mutants of human chorionic gonadotropin lacking N-glycosyl chain in the $\alpha$-subunit.1.Mechanism for the differential action of the N-linked carbohydrates; Biochemistry 36 12355-12363

Sinha N, Mohan S, Lipschultz C A and Smith-Gill S J 2002 Electrostatics in protein binding and function; Curr. Protein Pept. Sci. 3 601-614
Sinha N and Smith Gill S J 2002 Protein structure to function via dynamics; Protein Pept. Lett. 9 367-377

Srilatha N S and Murthy G S 1996 Mapping of assembled epitopic regions of human chorionic gonadotropin reveals proximity of CTP $\alpha$ to the determinant loop $\beta 93-100 ;$ J. Biosci. 21 755-764

Srilatha N S and Murthy G S 2002 Study of dissociation of human chorionic gonadotropin monoclonal antibody complexes using nitrocellulose as an insoluble support; Curr. Sci. 78 1548-1552

Tamil Selvi P, Ashish B and Murthy G S 2002 Determination of thermodynamic parameters of antigen-antibody interaction from real time kinetic studies; Curr. Sci. 82 1442-1448

Troalen F, Bellet B H, Ghillani P, Puisieux A, Bohuon C J and Bidart J M 1988 Antigenic determinants on human chorionic gonadotropin $\alpha$ subunit. II. Immunochemical mapping by a monoclonal anti-peptide antibody; J. Biol. Chem. 263 1037010376

Venkatesh N, Nagaraja G and Murthy G S 1995 Analysis of a conformation specific epitope of the alpha subunit of human chorionic gonadotropin: Study using monoclonal antibody probes; Curr. Sci. 69 48-55

Venkatesh N and Murthy G S 1997a Immunochemical approach to the mapping of an assembled epitope of human chorionic gonadotropin: Proximity of CTP- $\alpha$ to the receptor binding region of the B-subunit; J. Immunol. Methods $\mathbf{2 0 2}$ 173-182

Venkatesh N and Murthy G S 1997b Stability of assembled epitopes of human chorionic gonadotropin to covalent modifications: Analysis using Monoclonal antibody probes; Biochem. Mol. Biol. Int. 42 853-863

Venkatesh N, Krishnaswamy S, Meuris S and Murthy G S 1999 Epitope analysis and molecular modelling reveals the topography of the C-terminal peptide of $\beta$ subunit of hCG; Eur. J. Biochem. 265 1061-1066

MS received 20 September 2004; accepted 25 April 2005

ePublication: 27 May 2005

Corresponding editor: VIDYANAND NANJUNDIAH 